\title{
THE JACOBSON RADICAL OF A PROPOSITIONAL THEORY
}

\author{
GIULIO FELLIN, PETER SCHUSTER, AND DANIEL WESSEL
}

A Contribution to Ninety Years of Glivenko's Theorem

\begin{abstract}
Alongside the analogy between maximal ideals and complete theories, the Jacobson radical carries over from ideals of commutative rings to theories of propositional calculi. This prompts a variant of Lindenbaum's Lemma that relates classical validity and intuitionistic provability, and the syntactical counterpart of which is Glivenko's Theorem. The Jacobson radical in fact turns out to coincide with the classical deductive closure. As a by-product we obtain a possible interpretation in logic of the axioms-as-rules conservation criterion for a multi-conclusion Scott-style entailment relation over a single-conclusion one.
\end{abstract}

§1. Introduction. Glivenko's theorem from 1929 says that if a propositional formula $\varphi$ is provable in classical logic, then its double negation $\neg \neg \varphi$ is provable in intuitionistic logic. In 1933 Gödel extended this to predicate logic, which move required to admit on the intuitionistic side the scheme of double negation shift. With Gödel's and Gentzen's negative translation in place of double negation, both from 1933, one can even get by with minimal logic in place of intuitionistic logic. More than one related proof translation saw the light of the day, e.g., Kolmogorov's (1925) and Kuroda's (1951).

Glivenko's theorem thus stood right at the beginning of a fundamental change of perspective: that classical logic can be embedded into intuitionistic or even minimal logic, rather than the latter being a limited version of the former. Together with the revision of Hilbert's Programme ascribed to Kreisel and Feferman, this has led to the much broader quest for the computational content of classical proofs, today culminating in agile areas such as dynamical algebra, formal topology, program extraction from proofs, proof analysis, proof mining and proof translations. The growing success of these approaches suggests that customary mathematics, with

Received June 11, 2019.

2020 Mathematics Subject Classification. 03F03, 03F65.

Key words and phrases. Jacobson radical, propositional theory, Lindenbaum's Lemma, Glivenko's Theorem, axioms as rules, conservation criterion, entailment relation.

This note emerged from the the first and third author's M.Sc and Ph.D thesis, respectively $[38,113]$. For a preprint superseded by the present note see [40].

(C) The Author(s), 2021. Published by Cambridge University Press on behalf of The Association for Symbolic Logic. This is an Open Access article, distributed under the terms of the Creative Commons Attribution licence (https://creativecommons.org/licenses/by/4.0/), which permits unrestricted re-use, distribution, and reproduction in any medium, provided the original work is properly cited. 
classical logic and set theory, might eventually prove to be much more constructive than widely thought.

In 1930 Tarski ascribed to Lindenbaum the theorem that in classical logic any given theory $T$ equals the intersection of all the complete theories containing $T$. Its typical use for Gödel's Completeness Theorem aside, this Lindenbaum Lemma is one of several theorems from that period which describe the intersection of all the ideal objects extending a given concrete object. Those intersection theorems, in their full generality recognised as forms of the Axiom of Choice (AC), are often put by contraposition as extension or separation theorems. Apart from Lindenbaum's, prominent cases are known by the names of Artin-Schreier, Hahn-Banach, Krull and Szpilrajn. The case in algebra closest to Lindenbaum's Lemma, however, gained prominence only in 1945, when Jacobson pointed out the relevance of the intersection of all the maximal ideals of a given ring, i.e., of what is now known as the Jacobson radical.

In the present note we follow the analogy between maximal (proper) ideals and complete (consistent) theories to carry over the Jacobson radical from ideals of commutative rings to theories of propositional calculi (Section 5.2), where it turns out to coincide with the stable closure or with the closure with respect to classical logic (Proposition 3 and Corollary 1). This prompts a variant of Lindenbaum's Lemma that relates classical validity and intuitionistic provability (Proposition 2), and the syntactical counterpart of which happens to be Glivenko's Theorem in the form recalled above (Theorem 2).

As a by-product we obtain a possible interpretation in logic (Theorem 3) of the axioms-as-rules conservation criterion (Theorem 1) for a multiconclusion Scott-style entailment relation $\vdash$ over a single-conclusion one $\triangleright$. This criterion has proved to be the common core of many a syntactical counterpart of a semantic conservation theorem corresponding to one of the aforementioned intersection theorems. Typically any such case of conservation means reduction to a special case characterised by additional axioms with (possibly empty) disjunctions in positive position. Applying the criterion means to eliminate the additional axioms for $\vdash$ by way of the corresponding disjunction elimination rules for $\triangleright$. The latter equally suffice for proof practice, and have proved admissible in all mathematical instances yet considered.

Our interpretation of the conservation criterion in propositional logic (Theorem 3) is tantamount to Glivenko's Theorem (Theorem 2). As for the latter, disjunction elimination plays a central role in the proof of the former, together with some notorious features of (double) negation in intuitionistic logic and of provability in classical propositional logic (Lemmas 1 and 2).

§2. Preliminaries. Unless specified otherwise, we work in a suitable fragment of Aczel's Constructive Zermelo-Fraenkel Set Theory (CZF) [1-5] based on intuitionistic first-order predicate logic. While in general the concepts of this paper are elementary and the proofs are direct anyway, 
we still pin down CZF as metatheory if only rather for convenience's sake; in fact much less might suffice. Likewise, when we occasionally need to invoke a fragment of the principle of Excluded Middle or even a form of the AC, and thus go beyond $\mathbf{C Z F}$, we simply switch to $\mathbf{Z F}$ and $\mathbf{Z F C}$, respectively, and indicate this accordingly.

For example, the Restricted Law of Excluded Middle (REM) is not a principle of CZF. This REM means $\varphi \vee \neg \varphi$ for every set-theoretic formula $\varphi$ that is, bounded in the sense that only set-bounded quantifiers of the types $\forall x \in y$ and $\exists x \in y$ occur in $\varphi$. As is common in this context, negation is a defined connective: $\neg \varphi \equiv \varphi \rightarrow \perp$.

By a finite set we understand a set that can be written as $a_{1}, \ldots, a_{n}$ for some $n \geq 0$. Given any set $S$, let $\operatorname{Pow}(S)$ (respectively, $\operatorname{Fin}(S)$ ) consist of the (finite) subsets of $S$. We refer to [92] for further provisos to carry over to the present note. ${ }^{1}$

Convention. By an intermediate logic we mean an intermediate propositional calculus obtained by adding to the axioms of intuitionistic logic some classically valid propositional formulas [37].

We write $\triangleright$ to denote (deducibility in) any such intermediate logic in a propositional language $S$.

The subsequent properties of (double) negation are due to Brouwer for intuitionistic logic $[11,12,45,109,110]$ and carry over to an arbitrary $\triangleright$ :

Lemma 1. For any given intermediate logic $\triangleright$ in a propositional language $S$,

$$
\overline{\neg \neg \neg \psi \triangleright \neg \psi} \quad \frac{\Gamma, \varphi \triangleright \neg \psi}{\Gamma, \neg \neg \varphi \triangleright \neg \psi} \quad \overline{\triangleright \neg \neg(\psi \vee \neg \psi),}
$$

for every $\Gamma \in \operatorname{Pow}(S)$ and all $\varphi, \psi \in S$.

We refer to [55] and [77, p. 27] for a deeper discussion with earlier references of the following:

LEMMA 2. Let $\vdash$ and $\triangleright$ stand for classical logic and an intermediate logic, respectively, in a propositional language $S$. If $\Gamma \in \operatorname{Pow}(S)$ and $\psi \in S$, then $\Gamma \vdash \psi$ if and only if $\Gamma, \Delta \triangleright \psi$ for a suitable finite subset $\Delta$ of

$\operatorname{TND}_{0}(\Gamma, \psi)=\{\varphi \vee \neg \varphi: \varphi$ propositional variable occurring in $\Gamma$ or $\psi\}$, i.e., the set of relevant instances of tertium non datur for propositional variables.

A theory of an intermediate logic $\triangleright$ is a subset $T$ of the underlying propositional language $S$ that is, deductively closed with respect to $\triangleright$ :

$$
\forall \varphi \in S(T \triangleright \varphi \Rightarrow T \ni \varphi) .
$$

As usual, a theory $T$ of $\triangleright$ is

\footnotetext{
${ }^{1}$ For example, we deviate from the terminology prevalent in constructive mathematics and set theory $[4,5,8,9,64,69]$ : to reserve the term 'finite' to sets which are in bijection with $\{1, \ldots, n\}$ for a necessarily unique $n \geq 0$. Those exactly are the sets which are finite in our sense and are discrete too, i.e., have decidable equality [69].
} 
- consistent if $\perp \notin T$, which is to say that $T \neq S$;

- complete if

$$
\forall \varphi \in S(T \ni \varphi \vee \neg T \ni \varphi)
$$

- stable if

$$
\forall \varphi \in S(T \ni \neg \neg \varphi \Rightarrow T \ni \varphi)
$$

As an immediate consequence of Lemmas 1 and 2 we have the following:

Lemma 3. Let $\triangleright$ be an intermediate logic in a propositional language $S$. The following statements are equivalent for any given subset $T$ of $S$ :

1. $T$ is deductively closed with respect to classical logic.

2. $T$ is a stable theory of $\triangleright$.

3. $T$ is a theory of $\triangleright$ that contains all instances of excluded middle $\varphi \vee \neg \varphi$ with $\varphi \in S$.

4. $T$ is a theory of $\triangleright$ that contains all $\varphi \vee \neg \varphi$ where $\varphi$ is a propositional variable of $S$.

In particular, if a theory $T$ of an intermediate logic $\triangleright$ is complete, then $T$ is stable.

§3. Entailment relations. Entailment relations, both in their single- and multi-conclusion variant, are at the heart of this note. We briefly recall the basic notions, to which end we closely follow [91, 92].

3.1. Consequence. Let $S$ be a set and $\triangleright \subseteq \operatorname{Pow}(S) \times S$. Once abstracted from the context of logical formulas, all but one of Tarski's axioms of consequence [106] can be put as

$$
\frac{U \ni a}{U \triangleright a}(\mathrm{R}) \quad \frac{\forall b \in U(V \triangleright b) \quad U \triangleright a}{V \triangleright a}(\mathrm{~T}) \quad \frac{U \triangleright a}{\exists U_{0} \in \operatorname{Fin}(U)\left(U_{0} \triangleright a\right)}
$$

where $U, V \subseteq S$ and $a \in S$. These axioms also characterise a finitary covering or Stone covering in formal topology [95]; ${ }^{2}$ see further [17, 19, $72,73,97,98]$. The notion of consequence has allegedly been described first by Hertz [49-51]; see also [7, 59].

We do not employ the one of Tarski's axioms by which he required that $S$ be countable. This aside, Tarski has rather characterised the set of consequences of a set of propositions, which corresponds to the algebraic closure operator $U \mapsto U^{\triangleright}$ on $\operatorname{Pow}(S)$ of a relation $\triangleright$ as above where

$$
U^{\triangleright} \equiv\{a \in S: U \triangleright a\} .
$$

3.2. Single-conclusion entailment. Rather than with Tarski's notion, we henceforth work with its (tantamount) restriction to finite subsets, i.e., a

\footnotetext{
${ }^{2}$ This is from where we have taken the symbol $\triangleright$, used also $[16,112]$ to denote a 'consecution' [88].
} 
single-conclusion entailment relation. This is, a relation $\triangleright \subseteq \operatorname{Fin}(S) \times S$ such that

$$
\frac{U \ni a}{U \triangleright a}(\mathrm{R}) \quad \frac{V \triangleright b \quad V^{\prime}, b \triangleright a}{V, V^{\prime} \triangleright a}(\mathrm{~T}) \quad \frac{U \triangleright a}{U, U^{\prime} \triangleright a}(\mathrm{M})
$$

for all finite $U, U^{\prime}, V, V^{\prime} \subseteq S$ and $a, b \in S$, where as usual $U, V \equiv U \cup V$ and $V, b \equiv V \cup\{b\}$. Our focus thus is on finite subsets of $S$, for which we henceforth reserve the letters $U, V, W, \ldots$; we also sometimes write $a_{1}, \ldots, a_{n}$ in place of $\left\{a_{1}, \ldots, a_{n}\right\}$ even if $n=0$. Redefining

$$
T^{\triangleright} \equiv\{a \in S: \exists U \in \operatorname{Fin}(T)(U \triangleright a)\},
$$

for arbitrary subsets $T$ of $S$ gives back an algebraic closure operator on $\operatorname{Pow}(S)$. By writing $T \triangleright a$ in place of $a \in T^{\triangleright}$, the single-conclusion entailment relations thus correspond exactly to the relations satisfying Tarski's axioms above.

3.3. Multi-conclusion entailment. Let $S$ be a set and $\vdash \subseteq \operatorname{Fin}(S) \times$ Fin $(S)$. Scott's [102] axioms of entailment can be put as

$$
\frac{U \downarrow W}{U \vdash W}(\mathrm{R}) \quad \frac{V \vdash W, b \quad V^{\prime}, b \vdash W^{\prime}}{V, V^{\prime} \vdash W, W^{\prime}}(\mathrm{T}) \quad \frac{U \vdash W}{U, U^{\prime} \vdash W, W^{\prime}}
$$

for finite $U, U^{\prime}, V, V^{\prime}, W, W^{\prime} \subseteq S$ and $b \in S$, where $U \varnothing W$ means that $U$ and $W$ have an element in common [97]. Any such $\vdash$ is a multi-conclusion entailment relation, where 'multi' includes 'empty'. In practice, $\triangleright$ and $\vdash$ are inductively generated by the axioms of the intended models, which procedure we here take for granted [14, 31]; see also [3, 87, 92, 94].

This fairly general notion of entailment has been introduced by Scott [101-103], building on Hertz's and Tarski's work (see above), and of course on Gentzen's sequent calculus [43, 44]. Shoesmith and Smiley [104] trace multi-conclusion entailment relations back to Carnap [13], and Popper apparently had related ideas [85, 86]. ${ }^{3}$ Before Scott, Lorenzen had developed analogous concepts formally [65-68]; he even listed [66, pp. 8485] counterparts of the axioms $(\mathrm{R}),(\mathrm{T})$ and $(\mathrm{M})$ for single- and multiconclusion entailment $[27,81] .{ }^{4}$ As compared with Gentzen's and Lorenzen's approaches, Scott's entailment relation follow the contexts-as-sets paradigm, which has caused reservations [78, 79]. The relevance of the notion of entailment relation to point-free topology and constructive algebra has been pointed out in [14], and has been used very widely, e.g., in [20-22, 24, 25, 29, 32, 80, 89, 93, 100, 114, 115]. Consequence and entailment have further caught interest from various other angles [6, 35, 41, 52-54, 83, 99, 104, 117].

§4. Conservation. Again following [91, 92], we sketch the concept of conservative extension of a multi-conclusion entailment relation $\vdash$ over

\footnotetext{
${ }^{3}$ David Binder has kindly hinted us at Popper's work.

${ }^{4}$ Stefan Neuwirth has kindly pointed this out to us.
} 
a single-conclusion entailment relation $\triangleright$ on the same set $S$. After that we extract from [90] — based on [18] — possible interpretations limited to classical logic.

4.1. Conservation in syntax and semantics. Let $S$ be a set, and let $a, b, c, \ldots$ and $U, V, W, \ldots$ range over the elements of $S$ and $\operatorname{Fin}(S)$, respectively. Given a multi-conclusion entailment relation $\vdash$ and a single-conclusion entailment relation $\triangleright$ on the same set $S$, we throughout assume Extension:

$$
\text { Ext } \frac{U \triangleright a}{U \vdash a}
$$

Of major interest to us is the converse, alias Conservation:

$$
\text { Con } \frac{U \vdash a}{U \triangleright a}
$$

The trace of any given $\vdash$ is the single-conclusion entailment relation $\triangleright_{\vdash}$ defined by

$$
U \triangleright_{\vdash} a \equiv U \vdash a,
$$

for which Ext and Con are tantamount to $\triangleright \subseteq D_{\vdash}$ and $\triangleright \supseteq D_{\vdash}$, respectively.

An arbitrary subset $P$ of $S$ is a model of $\vdash$ if

$$
P \supseteq U \Rightarrow V \varnothing P \quad \text { whenever } U \vdash V .
$$

The notion of model carries over to single-conclusion $\triangleright$ in the apparent manner, such that the models of $\triangleright$ are exactly the $P \in \operatorname{Pow}(S)$ which are closed under $\triangleright$, i.e., for which $P^{\triangleright}=P$. Let $\operatorname{Mod}(\vdash)$ and $\operatorname{Mod}(\triangleright)$ consist of the models of $\vdash$ and $\triangleright$, respectively. By Extension, $\operatorname{Mod}(\vdash) \subseteq \operatorname{Mod}(\triangleright)$, which in ZFC is equivalent to Extension [92, Lemma 9].

Now Con follows from the Generalised Krull-Lindenbaum (GKL) Lemma, viz.

$$
\text { GKL } \quad \forall P \in \operatorname{Mod}(\vdash)(P \supseteq U \Rightarrow a \in P) \Longrightarrow U \triangleright a,
$$

the converse of which holds by Extension. Again by Extension, GKL implies the Trace Completeness Theorem (TCT), viz.

$$
\text { TCT } \quad \forall P \in \operatorname{Mod}(\vdash)(P \supseteq U \Rightarrow a \in P) \Longrightarrow U \vdash a,
$$

the converse of which holds by the definition of a model of $\vdash$. This TCT is a fragment of AC that implies REM [92, Corollary 5]. ${ }^{5}$

In ZFC, GKL and Con are equivalent [92, Theorem 6]. In CZF we can make this more precise:

REMARK 1. In the presence of Ext, GKL is equivalent to the conjunction of Con and TCT.

In all, GKL is semantic conservation, and Con is its syntactical counterpart.

\footnotetext{
${ }^{5}$ The proof of [92, Proposition 4] goes equally through with TCT in place of full CT.
} 
4.2. Conservation in proof practice. In proof practice, GKL is useful for reductions to special cases, by making possible to use $\vdash$ in proofs about $\triangleright$, but GKL is of semantic nature, entails REM and requires some AC. In comparison, Con is equally sufficient for that kind of reduction, is syntactical and has elementary proofs. Many such cases are known in point-free topology such as locale theory and formal topology $[14,15,20$, $23,70,71]$; in constructive algebra, especially with dynamical methods [26, $33,61-64,116,118,119]$; and in the proof theory of order relations [78, 80]. Most of those cases concern algebra at large. But what about logic? One may think of Gentzen's classical multi-succedent sequent calculus as extending his intuitionistic single-succedent variant $[43,44,77,105]$. As we will see, this thought goes in the right direction.

A typical situation is as follows: Let the single-conclusion entailment relation $\triangleright$ on a set $S$ be generated by axioms. Then the multi-conclusion entailment relation $\vdash$ on the same set $S$ is generated by the axioms of $\triangleright$, of course with $\vdash$ in place of $\triangleright$, and by additional axioms

$$
a_{1}, \ldots, a_{k} \vdash b_{1}, \ldots, b_{\ell},
$$

where $k, \ell \geq 0$. In any such situation we say that $\vdash$ extends $\triangleright$, and list the additional axioms if needed. This is legitimate inasmuch as if $\vdash$ extends $\triangleright$, then Ext is satisfied. What about Con?

The following most versatile conservation criterion [91, 92], which in fact gathers together many of the cases of Con mentioned before, will also help to understand Con for logic:

THEOREM 1. Let $\vdash$ extend $\triangleright$ with certain additional axioms of the form

$$
a_{1}, \ldots, a_{k} \vdash b_{1}, \ldots, b_{\ell},
$$

where $k, \ell \geq 0$. Then $\vdash$ and $\triangleright$ satisfy Con if and only if

$$
\frac{W, b_{1} \triangleright c \quad \cdots \quad W, b_{\ell} \triangleright c}{W, a_{1}, \ldots, a_{k} \triangleright c}
$$

for every additional axiom (2), all $c \in S$ and every $W \in \operatorname{Fin}(S)$.

This swiftly follows [92, Theorem 2] from a sandwich criterion for conservation given by Scott [102], and also is a corollary of cut elimination for entailment relations [94] as related to cut elimination in the presence of axioms [76].

Quite a few instances of GKL can be classified by the two cases named Universal Krull (UK) and Universal Lindenbaum (UL) in [92], for which $S$ is a set with

UK : a distinguished element $e$ of $S$ and a binary operation $*$ on $S$.

UL : a unary operation $\sim$ on $S$.

The additional axioms for $\vdash$ extending $\triangleright$ are

$$
\begin{array}{ll}
\mathrm{UK}: & e \vdash \quad a * b \vdash a, b, \\
\mathrm{UL}: & a, \sim a \vdash \vdash a, \sim a,
\end{array}
$$


where $a, b \in S$. The corresponding conservation criteria (Theorem 1) read

$$
\begin{array}{cl}
\mathrm{UK}: & \overline{W, e \triangleright c} \quad \frac{W, a \triangleright c \quad W, b \triangleright c}{W, a * b \triangleright c} \\
\mathrm{UL}: & \overline{W, a, \sim a \triangleright c} \quad \frac{W, a \triangleright c \quad W, \sim a \triangleright c}{W \triangleright c}
\end{array}
$$

where $W \in \operatorname{Fin}(S)$ and $a, b, c \in S .{ }^{6}$ We refer to [91, 92] for details and references.

4.3. The case of classical logic. Building upon [18], in [90] the instances of GKL in the cases UK and UL have been considered for the following data: $S$ consists of the sentences of a logical language, $\triangleright$ stands for deducibility with classical logic, $e$ is absurdity $\perp$, the operator $*$ is disjunction $\vee$, and $\sim$ is negation $\neg$. While the models of $\triangleright$ are the stable theories of $\triangleright$, the models of $\vdash$ are the complete consistent theories in $S$. Hence GKL is Lindenbaum's Lemma [106], and Con is provable but little interesting, simply because $\triangleright$ is classical logic already. Let's try to get more by relativising $\triangleright$.

Now let $\triangleright$ denote (deducibility in) an intermediate logic in a propositional language $S$; whence the models of $\triangleright$ are the theories of $\triangleright .{ }^{7}$ Let $\vdash$ extend $\triangleright$ with the following additional axioms:

$$
\perp \vdash \quad \vdash \varphi, \neg \varphi \quad(\varphi \in S) .
$$

The models of $\vdash$ are exactly the complete consistent theories of $\triangleright$, and the corresponding conservation criteria (Theorem 1 ) read

$$
\overline{\Gamma, \perp \triangleright \psi} \quad \frac{\Gamma, \varphi \triangleright \psi \quad \Gamma, \neg \varphi \triangleright \psi}{\Gamma \triangleright \psi}
$$

with $\Gamma \in \operatorname{Fin}(S)$ and $\varphi, \psi \in S$. While the first criterion holds for any given intermediate logic $\triangleright$, the second one amounts to $\triangleright$ satisfying $\triangleright \varphi \vee \neg \varphi$, which is to say that $\triangleright$ be classical logic. Hence Con in this case simply means that conservatively adding $\vdash \varphi, \neg \varphi$ is equivalent to requiring $\triangleright \varphi \vee \neg \varphi$. This of course is well known and of relatively little interest either. Can’t we do better?

\section{§5. Jacobson radicals.}

5.1. The Jacobson radical in algebra. Let $S=R$ be a commutative ring with 1 , and let $\triangleright$ stand for generation in $R$, i.e., $U \triangleright a$ means that $a$ is a linear combination with coefficients from $R$ of the elements of $U$. A model of $\triangleright$ is nothing but an ideal of $R$, i.e., a subset closed under linear combination. An ideal $J$ of $R$ is

- proper if $1 \notin J$, which is tantamount to $J \neq R$ and

\footnotetext{
${ }^{6}$ The criteria for UK have occurred [90] as ' $e$ is convincing for $\triangleright$ ' and ' $\triangleright$ satisfies Encoding'.

${ }^{7}$ For the related covering of formulas [30,96], the saturated sets rather are the complements of the theories.
} 
- complete if modulo $J$ any given $r \in R$ is either 0 or invertible, that is,

$$
\forall r \in R(J \ni r \vee J, r \triangleright 1) .
$$

Every proper complete ideal is a maximal ideal, i.e., maximal among the proper ideals, and vice versa in $\mathbf{Z F}$. With the current notation, the Jacobson radical of an ideal $J$ can be defined as

$$
\operatorname{Jac}(J)=\{a \in R: \forall b \in R(a, b \triangleright 1 \Rightarrow J, b \triangleright 1)\} .
$$

We thus carry over to commutative rings the first-order definition of the Jacobson radical for distributive lattices $[10,28,58]$ rather than using the more common one for commutative rings present e.g., in [64]. The latter reads

$$
\operatorname{Jac}(J)=\{a \in R: \forall b \in R \exists c \in R(1-(1-a b) c \in J)\},
$$

which is to say that any given $a \in R$ belongs to $\operatorname{Jac}(R)$ precisely when $1-a b$ is invertible modulo $J$ for every $b \in R$. We give precedence to (5) over (6) because the former, unlike the latter, can be transferred to logic without further ado (Section 5.2).

Just as (6), the first-order definition we employ (5) is anyway equivalent in $\mathbf{Z F C}$ to the following more customary second-order characterisation of the Jacobson radical [57]. Although the proof is of course similar to the one with (6) in place of (5) and for maximal rather than complete ideals, see e.g., [64], we detail this one because it carries over to logic (Proposition 2).

Proposition 1 (ZFC). For every ideal $J$ of a commutative ring $R$,

$$
\bigcap \operatorname{Com}_{J}(R)=\operatorname{Jac}(J)
$$

where $\operatorname{Com}_{J}(R)$ consists of the complete ideals $\mathrm{c}$ in $R$ with $J \subseteq \mathrm{c}$.

Proof. Let $a \in \operatorname{Jac}(J)$, and let $\mathrm{c}$ be a complete ideal such that $\mathrm{c} \supseteq J$. Either $\mathrm{c} \ni a$ or c, $a \triangleright 1$. In the former case we are done. In the latter case there is $b \in R$ such that $\mathrm{c} \triangleright b$ (in particular, $b \in \mathrm{c}$ ) and $a, b \triangleright 1$. Since $a \in \operatorname{Jac}(J)$, we get $J, b \triangleright 1$. As $J \subseteq \mathrm{c}$ and $b \in \mathrm{c}$, this implies $\mathrm{c} \triangleright 1$. Hence $\mathrm{c}=R$, by which again $\mathrm{c} \ni a$.

Conversely, if $a \notin \operatorname{Jac}(J)$, then there is $b \in R$ for which $a, b \triangleright 1$ holds but $J, b \triangleright 1$ fails, and thus $(J, b)^{\triangleright}$ lacks $a$. Zorn's Lemma yields an ideal c maximal among the ones that contain $(J, b)^{\triangleright}$ yet miss $a$. Any such $\mathrm{c}$ is complete: if $\mathrm{c} \not \supset b$, then $\mathrm{c}, b \triangleright a$ by maximality, and thus $\mathrm{c}, b \triangleright 1$ by $a, b \triangleright 1$.

It obviously is irrelevant whether the intersection ranges over the only improper ideal $R$ as well.

5.2. The Jacobson radical in logic. Let again $\triangleright$ stand for (deducibility in) an intermediate logic in a propositional language $S$. That a (consistent) theory $T$ of $\triangleright$ be complete can equivalently be put as

$$
\forall \varphi \in S(T \ni \varphi \vee T, \varphi \triangleright \perp) \text {. }
$$


This move makes fully evident the analogy between complete ideals (4) and complete theories (7), which rests upon the following brief (and necessarily incomprehensive) dictionary:

\begin{tabular}{ll}
\hline propositional logic & commutative algebra \\
\hline language & ring \\
deduction $\triangleright$ & generation $\triangleright$ \\
theory & ideal \\
absurdity $\perp$ & unit 1 \\
consistent theory & proper ideal \\
complete theory & complete ideal \\
\hline
\end{tabular}

With this at hand we translate (5) into a definition of the Jacobson radical of a theory $T$ :

$$
\operatorname{Jac}(T)=\{\alpha \in S: \forall \beta \in S(\alpha, \beta \triangleright \perp \Rightarrow T, \beta \triangleright \perp)\} .
$$

This is obviously equivalent to the following characterisation:

$$
\operatorname{Jac}(T)=\{\alpha \in S: \forall \beta \in S(\alpha \triangleright \neg \beta \Rightarrow T \triangleright \neg \beta)\} .
$$

Mutatis mutandis the proof of Proposition 1 proves what we would like to provisionally call the Intermediate Lindenbaum Lemma:

Proposition 2 (ZFC). For every theory $T$ of an intermediate logic $\triangleright$ in a propositional languageS,

$$
\text { ILL } \bigcap \operatorname{Com}_{T}(S)=\operatorname{Jac}(T),
$$

where $\operatorname{Com}_{T}(S)$ consists of the complete (consistent) theories $C$ in $S$ with $T \subseteq C$.

As for Proposition 1, it is irrelevant whether the intersection includes the only inconsistent theory $S$.

Since every complete theory is stable (Lemma 3), the left-hand side of ILL is as for the original Lindenbaum Lemma [106] in the form

$$
\bigcap \operatorname{Com}_{T}(S)=T \text {, }
$$

for every stable theory $T$ in $S$. Hence the left-hand side of ILL equals in ZFC the classical deductive closure of $T$. What about the right-hand side of ILL?

Proposition 3. For every theory $T$ of an intermediate logic $\triangleright$ in a propositional language $S$,

$$
\operatorname{Jac}(T)=\{\alpha \in S: T \ni \neg \neg \alpha\} .
$$

Proof. Let $\alpha \in \operatorname{Jac}(T)$. Since $\alpha \triangleright \neg \neg \alpha$, we get $T \triangleright \neg \neg \alpha$. Conversely, let $\alpha \in S$ be such that $T \ni \neg \neg \alpha$. If $\beta \in S$ is such that $\alpha \triangleright \neg \beta$, then $\neg \neg \alpha \triangleright \neg \beta$ and thus $T \triangleright \neg \beta$. 
With Lemma 3 at hand we obtain the following:

COROLlary 1. $\operatorname{Jac}(T)$ is the least stable theory of $\triangleright$ which contains the given theory $T$ of $\triangleright$; in other words, $\operatorname{Jac}(T)$ equals the deductive closure of $T$ with respect to classical logic.

So ILL for any intermediate logic $\triangleright$ whatsoever is nothing but the original Lindenbaum Lemma!

Now let $\triangleright$ be intuitionistic logic $\triangleright_{i}$, and write $\triangleright_{c}$ for classical logic, always in the given propositional language $S$. In this case and by the above, Lindenbaum's Lemma in the form of ILL is the semantics of Glivenko's Theorem [46], which in turn is well known as purely syntactical:

Theorem 2 (Glivenko 1929). Let $S$ be a propositional language. For all $\Gamma \in \operatorname{Fin}(S)$ and $\varphi \in S$,

$$
\Gamma \triangleright_{c} \varphi \Rightarrow \Gamma \triangleright_{i} \neg \neg \varphi .
$$

For example, this follows from Corollary 1. We hasten to add that the latter rests upon Lemmas 1 and 2, which of course are the main ingredients of a very common proof of Glivenko's theorem. Recent literature about Glivenko's Theorem includes [36, 39, 42, 48, 56, 60, 74, 75, 82, 84]. ${ }^{8}$

§6. Glivenko's theorem as syntactical conservation. Once more let $\triangleright_{i}$ and $\triangleright_{c}$ stand for intuitionistic and classical logic in a propositional language $S$. For $\Gamma, \Delta \in \operatorname{Fin}(S)$ and $\varphi \in S$, set

$$
\Gamma \triangleright_{g} \varphi \equiv \Gamma \triangleright_{i} \neg \neg \varphi \quad \text { and } \quad \Gamma \vdash_{c} \Delta \equiv \Gamma \triangleright_{c} \bigvee \Delta,
$$

which defines a single- and a multi-conclusion entailment relation, respectively. Of course the trace of $\vdash_{c}$ is nothing but $\triangleright_{c}$; so Glivenko's Theorem (Theorem 2) can be rephrased as the following syntactical conservation:

THEOREM 3. The extension $\vdash_{c}$ of $\triangleright_{g}$ is conservative, that is,

$$
\text { Gli } \Gamma \triangleright_{c} \varphi \Rightarrow \Gamma \triangleright_{g} \varphi,
$$

for all $\Gamma \in \operatorname{Fin}(S)$ and $\varphi \in S$.

To see how Theorem 1 applies in this context, we now prove Theorem 3 in detail. Clearly this proof will otherwise have the main ingredients of any proof of Glivenko's Theorem (Theorem 2). By Lemma 2, $\vdash_{c}$ extends $\triangleright_{i}$, and thus $\triangleright_{g}$, with the following additional axioms:

$$
\perp \vdash_{c} \quad \vdash_{c} \varphi, \neg \varphi \quad(\varphi \in S) .
$$

The corresponding conservation criteria (3) read

$$
\overline{\Gamma, \perp \triangleright_{g} \psi} \quad \frac{\Gamma, \varphi \triangleright_{g} \psi \quad \Gamma, \neg \varphi \triangleright_{g} \psi}{\Gamma \triangleright_{g} \psi}
$$

with $\Gamma \in \operatorname{Fin}(S)$ and $\varphi, \psi \in S$.

\footnotetext{
${ }^{8}$ This list is by no means meant exhaustive.
} 
To prove Theorem 3, in view of Theorem 1 it thus is (necessary and) sufficient to verify (9). Writing $\triangleright_{i} \neg \neg$ for $\triangleright_{g}$ this goes as follows. Needless to say, $\Gamma, \perp \triangleright_{i} \neg \neg \psi$. If both $\Gamma, \varphi \triangleright_{i} \neg \neg \psi$ and $\Gamma, \neg \varphi \triangleright_{i} \neg \neg \psi$, then $\Gamma, \varphi \vee \neg \varphi \triangleright_{i} \neg \neg \psi$ by disjunction elimination. By Lemma 1 we get $\Gamma, \neg \neg(\varphi \vee \neg \varphi) \triangleright_{i} \neg \neg \psi$ and thus $\Gamma \triangleright_{i} \neg \neg \psi$ as desired.

As the models of $\vdash_{c}$ are exactly the complete consistent theories, ILL for $T \equiv \Gamma^{\triangleright_{i}}$ with $\Gamma \in \operatorname{Fin}(S)$ is to Gli just as GKL is to Con for $\vdash \equiv \vdash_{c}$ and $\triangleright \equiv \triangleright_{g}$. Although $\vdash_{c}$ equally extends $\triangleright_{i}$ with the same additional axioms (8), and the first conservation criterion of (9) also holds for $\triangleright_{i}$ in place of $\triangleright_{g}$, this of course is not the case in general for the second one, e.g., if $\psi \equiv \varphi \vee \neg \varphi$.

We conclude by a relativised version of Glivenko's Theorem as syntactical conservation. Let $\Gamma \subseteq \operatorname{Fin}(S)$ and $\psi \in S$. With $\operatorname{TND}_{0}(\Gamma, \psi)$ as in Lemma 2, for every propositional variable $\varphi \in S$ consider the conservation criterion from Theorem 1 for $\vdash_{c} \varphi, \neg \varphi$ over $\triangleright_{i}$ :

$$
\mathrm{Cri}_{\varphi}(\Gamma, \psi): \frac{\Gamma, \Delta, \varphi \triangleright_{i} \psi \quad \Gamma, \Delta, \neg \varphi \triangleright_{i} \psi}{\Gamma, \Delta \triangleright_{i} \psi} \text { or, equivalently, } \frac{\Gamma, \Delta, \varphi \vee \neg \varphi \triangleright_{i} \psi}{\Gamma, \Delta \triangleright_{i} \psi}
$$

Proposition 4. For arbitrary but fixed $\Gamma \subseteq \operatorname{Fin}(S)$ and $\psi \in S$, the following items are equivalent:

1. $\operatorname{Cri}_{\varphi}(\Gamma, \psi)$ for all propositional variables $\varphi \in S$ occurring in $\Gamma$ or $\psi$.

2. $\Gamma \triangleright_{c} \psi \Rightarrow \Gamma \triangleright_{i} \psi$, i.e.,,$\vdash_{c}$ is conservative over $\triangleright_{i}$ for the given $\Gamma$ and $\psi$.

While the first implies the second item by Lemma 2 and Theorem 1, the converse is evident.

Glivenko's Theorem 2 is the case of the second item in which $\Gamma$ is arbitrary but $\psi$ is a negated formula, in which case the first item obtains by Lemma 1 . Other cases include the one in which $\Gamma \cup\{\psi\}$ is made of negative formulas only; see e.g., [34, 107, 108].

§7. Complements. We briefly review some related observations recently made about double negation [39] in the more general context of a nucleus $j$ in place of $\neg \neg$.

First, for any given intermediate propositional logic $\triangleright$ the following are equivalent:
A. $\Gamma \vdash_{c} \varphi \Rightarrow \Gamma \triangleright \neg \neg \varphi$ for all $\Gamma, \varphi$ and
B. $\varphi \rightarrow \neg \neg \psi \triangleright \neg \neg(\varphi \rightarrow \psi)$ for all $\varphi, \psi$.

Now B is well-known to hold whenever $\triangleright$ is intuitionistic logic $\triangleright_{i}$ (see, e.g., [111, Lemma 6.2.2]), in which case A becomes Glivenko's theorem. $A$ posteriori B holds for any intermediate logic $\triangleright$ whatsover, as any such $\triangleright$ extends $\triangleright_{i}$.

Next, if $\triangleright$ is an intermediate predicate logic, then $\mathrm{A}$ is equivalent to B in conjunction with the double negation shift for $\triangleright$ :

C. $\forall x \neg \neg \varphi \triangleright \neg \neg \forall x \varphi$ for all formulae $\varphi$. 
Again if $\triangleright$ is intuitionistic logic $\triangleright_{i}$, this yields Gödel's extension of Glivenko's theorem [37, 47].

Now $\mathrm{C}$ trivially holds for any existential logic, i.e., without $\forall$ altogether, for which A with $\triangleright_{i}$ as $\triangleright$ is [107, Corollary to Proposition 2.3.8]. For A to hold it suffices to refrain from using the $\forall$-introduction rule or right rule $\mathbf{R} \forall$, which in fact is the only rule that can cause issues in this setting [39]. To be able to avoid $\mathbf{R} \forall$ it is enough that the sequent under consideration have no positive occurrences of $\forall$, because derivations of such sequents by classical logic can be cleared from that rule (see, e.g., [74]).

Acknowledgments. The present study was carried out within the projects "A New Dawn of Intuitionism: Mathematical and Philosophical Advances" (ID 60842) funded by the John Templeton Foundation, and "Reducing complexity in algebra, logic, combinatorics-REDCOM" belonging to the programme "Ricerca Scientifica di Eccellenza 2018" of the Fondazione Cariverona. The opinions expressed in this paper are those of the authors and do not necessarily reflect the views of those foundations. An early version of the manuscript was conceived when the authors were visiting the Hausdorff Research Institute for Mathematics at Universität Bonn in 2018 during the Trimester Program "Types, Sets and Constructions." Useful hints by Sara Negri are gratefully acknowledged. Schuster was glad for the feedback he received at the Scuola estiva di logica 2019 in Gargnano.

\section{REFERENCES}

[1] P. ACZEL, The type theoretic interpretation of constructive set theory, Logic Colloquium '77, Studies in Logic and the Foundations of Mathematics, vol. 96, NorthHolland, Amsterdam, 1978, pp. 55-66.

[2] - The type theoretic interpretation of constructive set theory: Choice principles,

The L. E. J. Brouwer Centenary Symposium, Studies in Logic and the Foundations of Mathematics, vol. 110, North-Holland, Amsterdam, 1982, pp. 1-40.

[3] — The type theoretic interpretation of constructive set theory: Inductive definitions, Logic, Methodology and Philosophy of Science, VII, Studies in Logic and the Foundations of Mathematics, vol. 114, North-Holland, Amsterdam, 1986, pp. 17-49.

[4] P. Aczel and M. RathJen, Notes on constructive set theory, Technical report no. 40, Institut Mittag-Leffler, 2000.

[5] - Constructive set theory, Book draft, 2010. Available at https://www1.maths.leeds.ac.uk/ rathjen/book.pdf (accessed 24 August, 2021).

[6] A. Avron, Simple consequence relations. Information and Computation, vol. 92 (1991), pp. $105-139$.

[7] J.-Y. BÉZIAU, Les axiomes de Tarski, La philosophie en Pologne 1919-1939 (R. Pouivet and M. Resbuschi, editors), Librairie Philosophique J. VRIN, Paris, 2006.

[8] E. BisHop, Foundations of Constructive Analysis, McGraw-Hill, New York, 1967.

[9] E. Bishop and D. Bridges, Constructive Analysis, Springer, Berlin-Heidelberg, 1985.

[10] A. Blass, Prime ideals yield almost maximal ideals. Fundamenta Mathematicae, vol. 127 (1987), no. 1, pp. 57-66.

[11] L. E. J. Brouwer, De onbetrouwbaarheid der logische principes, Tijdschrift voor Wijsbegeerte, vol. 2 (1908), pp. 152-158.

[12] - Intuitionistische Zerlegung mathematischer Grundbegriffe, Jahresbericht der Deutschen Mathematiker-Vereinigung, vol. 33 (1925), pp. 251-256. 
[13] R. CARnAP, Formalization of Logic, Harvard University Press, Cambridge, 1943.

[14] J. Cederquist and T. CoquAND, Entailment relations and distributive lattices, Logic Colloquium '98 (S. R. Buss, P. Hájek, and P. Pudlák, editors), Lecture Notes in Logic, vol. 13, A. K. Peters, Natick, 2000, pp. 127-139.

[15] J. Cederquist, T. Coquand, and S. Negri, The Hahn-Banach theorem in type theory, Twenty-Five Years of Constructive Type Theory (G. Sambin and J. M. Smith, editors), Oxford Logic Guides, vol. 36, Oxford University Press, New York, 1998, pp. 57-72.

[16] P. Cintula and N. CARLES, The proof by cases property and its variants in structural consequence relations. Studia Logica, vol. 101 (2013), no. 4, pp. 713-747.

[17] F. Ciraulo, M. E. Maietti, and G. Sambin, Convergence in formal topology: A unifying notion. Journal of Logic and Analysis, vol. 5 (2013), no. 2, pp. 1-45.

[18] F. Ciraulo, D. Rinaldi, and P. Schuster, Lindenbaum's lemma via open induction, Advances in Proof Theory (R. Kahle, T. Strahm, and T. Studer, editors), Progress in Computer Science and Applied Logic, vol. 28, Springer, Cham, 2016, pp. 65-77.

[19] F. Ciraulo and G. Sambin, Finitary formal topologies and Stone's representation theorem. Theoretical Computer Science, vol. 405 (2008), nos. 1-2, pp. 11-23.

[20] T. CoQunand, A direct proof of the localic Hahn-Banach theorem, 2000. Available at http://www.cse.chalmers.se/ coquand/formal.html (accessed 24 August, 2021).

[21] —, Lewis Carroll, Gentzen and entailment relations, 2000. Available at http://www.cse.chalmers.se/ ${ }^{\sim}$ coquand/formal.html.

[22] - About Stone's notion of spectrum. Journal of Pure and Applied Algebra, vol. 197 (2005), nos. 1-3, pp. 141-158.

[23] — Geometric Hahn-Banach theorem. Mathematical Proceedings of the Cambridge Philosophical Society, vol. 140 (2006), pp. 313-315.

[24] - Space of valuations. Annals of Pure and Applied Logic, vol. 157 (2009), 97-109.

[25] T. Coquand and H. Lombardi, Hidden constructions in abstract algebra: Krull dimension of distributive lattices and commutative rings, Commutative Ring Theory and Applications (M. Fontana, S.-E. Kabbaj, and S. Wiegand, editors), Lecture Notes in Pure and Applied Mathematics, vol. 231, Addison-Wesley, Reading, 2002, pp. 477-499.

[26] - A logical approach to abstract algebra. Mathematical Structures in Computer Science, vol. 16 (2006), pp. 885-900.

[27] T. Coquand, H. Lombardi, and S. NeUWIRTH, Lattice-ordered groups generated by an ordered group and regular systems of ideals. The Rocky Mountain Journal of Mathematics, vol. 49 (2019), no. 5, pp. 1449-1489.

[28] T. Coquand, H. Lombardi, and C. Quitté, Dimension de Heitmann des treillis distributifs et des anneaux commutatifs. Publications Mathématiques de Besançon: Algèbre et Théorie des Nombres, 2006, pp. 57-100.

[29] T. Coquand and H. Persson, Valuations and Dedekind's Prague theorem. Journal of Pure and Applied Algebra, vol. 155 (2001), nos. 2-3, pp. 121-129.

[30] T. Coquand, S. Sadocco, G. Sambin, and J. M. Smith, Formal topologies on the set of first-order formulae. The Journal of Symbolic Logic, vol. 65 (2000), no. 3, pp. 1183-1192.

[31] T. Coquand, G. Sambin, J. Smith, and S. Valentini, Inductively generated formal topologies. Annals of Pure and Applied Logic, vol. 124 (2003), pp. 71-106.

[32] T. Coquand and G.-Q. Zhang, Sequents, frames, and completeness, Computer Science Logic (P. G. Clote and H. Schwichtenberg, editors), Lecture Notes in Computer Science, vol. 1862, Springer, Berlin, 2000, pp. 277-291.

[33] M. Coste, H. Lombardi, and M.-F. Roy, Dynamical method in algebra: Effective Nullstellensätze. Annals of Pure and Applied Logic, vol. 111 (2001), no. 3, pp. 203-256.

[34] R. David, K. Nour, and C. Raffalli, Introduction à la Logique. Théorie de la démonstration, second ed., Dunod, Paris, 2003.

[35] K. DošEn, On passing from singular to plural consequences, Logic at Work: Essays Dedicated to the Memory of Helena Rasiowa (E. Orlowska, editor), Studies in Fuzziness and Soft Computing, vol. 24, Physica, Heidelberg, 1999, pp. 533-547. 
[36] C. Espíndola, A short proof of Glivenko theorems for intermediate predicate logics. Archive for Mathematical Logic, vol. 52 (2013), nos. 7-8, pp. 823-826.

[37] Encyclopedia of Mathematics, Intermediate logic, 2016. Available at http://www.encyclopediaofmath.org/index.php?title=Intermediate_logic\&oldid=39747 (accessed 13 November, 2016).

[38] G. Fellin, The Jacobson Radical: From Algebra to Logic, Master's thesis, Università di Verona, Dipartimento di Informatica, Verona, 2018.

[39] G. FeLLIN and P. SChUSTER, A general Glivenko-Gödel theorem for nuclei, Proceedings of the 37th Conference on the Mathematical Foundations of Programming Semantics, MFPS 2021 (A. Sokolova, editor), Electronic Notes in Theoretical Computer Science, Elsevier, 2021.

[40] G. Fellin, P. Schuster, and D. Wessel, The Jacobson radical of a propositional theory, Proof-Theoretic Semantics: Assessment and Future Perspectives (T. Piecha and P. Schroeder-Heister, editors), University of Tübingen, 2019, pp. 287-299, http://doi.org/10.15496/publikation-35319.

[41] D. M. GabbaY, Semantical Investigations in Heyting's Intuitionistic Logic, Synthese Library, vol. 148, D. Reidel Publishing Co., Dordrecht-Boston, 1981.

[42] N. Galatos and H. Ono, Glivenko theorems for substructural logics over FL. The Journal of Symbolic Logic, vol. 71 (2006), no. 4, pp. 1353-1384.

[43] G. GentZEn, Untersuchungen über das logische Schließen I. Mathematische Zeitschrift, vol. 39 (1934), pp. 176-210.

[44] - Untersuchungen über das logische Schließen II. Mathematische Zeitschrift, vol. 39 (1934), pp. 405-431.

[45] V. Glivenko, Sur la logique de M. Brouwer. Academie Royale des Sciences des Lettres et des Beaux-Arts de Belgique. Bulletin de la Classe des Sciences Cinquième Série, vol. 14 (1928), pp. 225-228.

[46] _- Sur quelques points de la Logique de M. Brouwer. Academie Royale des Sciences des Lettres et des Beaux-Arts de Belgique. Bulletin de la Classe des Sciences Cinquième Série, vol. 15 (1929), 183-188.

[47] K. GöDEL, Über eine bisher noch nicht benützte Erweiterung des finiten Standpunktes. Dialectica, vol. 12 (1958), pp. 280-287.

[48] G. Guerrieri and A. Naibo, Postponement of raa and Glivenko's theorem, revisited. Studia Logica, vol. 107 (2019), no. 1, pp. 109-144.

[49] P. Hertz, Über Axiomensysteme für beliebige Satzsysteme. I. Teil. Sätze ersten Grades. Mathematische Annalen, vol. 87 (1922), no. 3, pp. 246-269.

[50] — Über Axiomensysteme für beliebige Satzsysteme. II. Teil. Sätze höheren Grades. Mathematische Annalen, vol. 89 (1923), no. 1, pp. 76-102.

[51] - Über Axiomensysteme für beliebige Satzsysteme. Mathematische Annalen, vol. 101 (1929), no. 1, pp. 457-514.

[52] L. Humberstone, On a conservative extension argument of Dana Scott. Logic Journal of the IGPL, vol. 19 (2011), pp. 241-288.

[53] - Dana Scott's work with generalized consequence relations, Universal Logic: An Anthology. From Paul Hertz to Dov Gabbay (J.-Y. Béziau, editor), Studies in Universal Logic, Birkhäuser, Basel, 2012, pp. 263-279.

[54] R. Iemhoff, Consequence relations and admissible rules. Journal of Philosophical Logic, vol. 45 (2016), no. 3, pp. 327-348.

[55] H. Ishinara, Classical propositional logic and decidability of variables in intuitionistic propositional logic. Logical Methods in Computer Science, vol. 10 (2014), no. 3, pp. 3:1-3:7.

[56] H. Ishihara and H. SChWichtenberg, Embedding classical in minimal implicational logic. Mathematical Logic Quarterly, vol. 62 (2016), nos. 1-2, pp. 94-101.

[57] N. JaCOBSON, The radical and semi-simplicity for arbitrary rings. American Journal of Mathematics, vol. 67 (1945), no. 2, pp. 300-320.

[58] P. Johnstone, Almost maximal ideals. Fundamenta Mathematicae, vol. 123 (1984), no. 3, pp. 197-209. 
[59] J. Legris, Paul Hertz and the origins of structural reasoning, Universal Logic: An Anthology. From Paul Hertz to Dov Gabbay (J.-Y. Béziau, editor), Studies in Universal Logic, Birkhäuser, Basel, 2012, pp. 3-10.

[60] T. LitaK, M. Polzer, and U. RabensteIn, Negative translations and normal modality, 2nd International Conference on Formal Structures for Computation and Deduction, LIPIcsLeibniz International Proceedings in Informatics, vol. 84, Schloss Dagstuhl - LeibnizZentrum für Informatik, Wadern, 2017, Article no. 27, pp. 27:1-27:18.

[61] H. LOMBARDI, Le contenu constructif d'un principe local-global avec une application à la structure d'un module projectif de type fini, Publications Mathématiques de Besançon: Algèbre et Théorie des Nombres, 1997, Fascicule 94-95 \& 95-96.1997

[62] - Relecture constructive de la théorie d'Artin-Schreier. Annals of Pure and Applied Logic, vol. 91 (1998), pp. 59-92.

[63] - Algèbre dynamique, espaces topologiques sans points et programme de Hilbert. Annals of Pure and Applied Logic, vol. 137 (2006), pp. 256-290.

[64] H. Lombardi and C. Quitté, Commutative Algebra: Constructive Methods. Finite Projective Modules, Algebra and Applications, vol. 20, Springer Netherlands, Dordrecht, 2015.

[65] P. LoRENZEN, Über halbgeordnete Gruppen. Mathematische Zeitschrift, vol. 52 (1950), no. 1 , pp. $483-526$.

[66] - Algebraische und logistische Untersuchungen über freie Verbände. The Journal of Symbolic Logic, vol. 16 (1951), no. 2, pp. 81-106.

[67] — Teilbarkeitstheorie in Bereichen. Mathematische Zeitschrift, vol. 55 (1952), no. 3, pp. 269-275.

[68] - Die Erweiterung halbgeordneter Gruppen zu Verbandsgruppen. Mathematische Zeitschrift, vol. 58 (1953), no. 1, pp. 15-24.

[69] R. Mines, F. Richman, and W. Ruitenburg, A Course in Constructive Algebra, Universitext, Springer, New York, 1988.

[70] C. J. Mulvey and J. Wick-Pelletier, The dual locale of a seminormed space. Cahiers de Topologie et Géométrie Différentielle Catégoriques, vol. 23 (1982), no. 1, pp. 73-92.

[71] - A globalization of the Hahn-Banach theorem. Advances in Mathematics, vol. 89 (1991), pp. $1-59$.

[72] S. Negri, Stone bases alias the constructive content of Stone representation, Logic and Algebra (A. Ursini and P. Aglianò, editors), Lecture Notes in Pure and Applied Mathematics, vol. 180, Marcel Dekker, New York, 1996, pp. 617-636.

[73] - Continuous domains as formal spaces. Mathematical Structures in Computer Science, vol. 12 (2002), no. 1, pp. 19-52.

[74] - Proof analysis beyond geometric theories: From rule systems to systems of rules. Journal of Logic and Computation, vol. 26 (2014), no. 2, pp. 513-537.

[75] - Glivenko sequent classes in the light of structural proof theory. Archive for Mathematical Logic, vol. 55 (2016), nos. 3-4, pp. 461-473.

[76] S. Negri and J. von Plato, Cut elimination in the presence of axioms, this Journal, vol. 4 (1998), no. 4, pp. 418-435.

[77] —, Structural Proof Theory, Cambridge University Press, Cambridge, 2001.

[78] _ Proof Analysis: A Contribution to Hilbert's Last Problem, Cambridge University Press, Cambridge, 2011.

[79] - Cut elimination in sequent calculi with implicit contraction, with a conjecture on the origin of Gentzen's altitude line construction, Concepts of Proof in Mathematics, Philosophy, and Computer Science (D. Probst and P. Schuster, editors), Ontos Mathematical Logic, vol. 6, Walter de Gruyter, Berlin, 2016, pp. 269-290.

[80] S. Negri, J. von Plato, and T. Coquand, Proof-theoretical analysis of order relations. Archive for Mathematical Logic, vol. 43 (2004), pp. 297-309. 
[81] S. NeuwirTh, Lorenzen's theory of divisibility in monoid-preordered sets, preprint, 2018. Available at https://lmb.univ-fcomte.fr/IMG/pdf/lorenzen_s_theory_of_ divisibility_in_monoid_preordered_sets.pdf.

[82] H. Ono, Glivenko theorems revisited. Annals of Pure and Applied Logic, vol. 161 (2009), no. 2, pp. 246-250.

[83] G. Payette and P. K. Sснотch, Remarks on the Scott-Lindenbaum theorem. Studia Logica, vol. 102 (2014), no. 5, pp. 1003-1020.

[84] L. C. Pereira and E. H. Haeusler, On constructive fragments of classical logic, Dag Prawitz on proofs and meaning, Outstanding Contributions to Logic, vol. 7, Springer, Cham, 2015, pp. 281-292.

[85] K. R. Popper, On the theory of deduction, Part I. Derivation and its generalizations. Proceedings of the Koninklijke Nederlandse Akademie van Wetenschappen, vol. 51 (1948), no. 2, pp. 173-183.

[86] - On the theory of deduction, Part II. The definitions of classical and intuitionist negation. Proceedings of the Koninklijke Nederlandse Akademie van Wetenschappen, vol. 51 (1948), no. 3, pp. 322-331.

[87] M. RATHJEN, Generalized inductive definitions in constructive set theory, From Sets and Types to Topology and Analysis: Towards Practicable Foundations for Constructive Mathematics (L. Crosilla and P. Schuster, editors), Oxford Logic Guides, vol. 48, Clarendon Press, Oxford, 2005, Chap. 16.

[88] G. ReSTALL, An Introduction to Substructural Logics, Routledge, London, 2000.

[89] D. Rinaldi, Formal Methods in the Theories of Rings and Domains. Doctoral dissertation, Universität München, 2014.

[90] D. Rinaldi and P. Schuster, A universal Krull-Lindenbaum theorem. Journal of Pure and Applied Algebra, vol. 220 (2016), pp. 3207-3232.

[91] D. Rinaldi, P. Schuster, and D. Wessel, Eliminating disjunctions by disjunction elimination. this JOURNAL, vol. 23 (2017), no. 2, pp. 181-200.

[92] - Eliminating disjunctions by disjunction elimination. Indagationes Mathematicae. New Series, vol. 29 (2018), no. 1, pp. 226-259.

[93] D. Rinaldi and D. Wessel, Extension by conservation. Sikorski's theorem. Logical Methods in Computer Science, vol. 14 (2018), nos. 4:8, pp. 1-17.

[94] - Cut elimination for entailment relations. Archive for Mathematical Logic, vol. 58 (2019), nos. 5-6, pp. 605-625.

[95] G. Sambin, Intuitionistic formal spaces - A first communication, Mathematical Logic and Its Applications (D. Skordev, editor), Plenum, New York, 1987, pp. 187-204.

[96] - Pretopologies and completeness proofs. The Journal of Symbolic Logic, vol. 60 (1995), no. 3, pp. 861-878.

[97] - Some points in formal topology. Theoretical Computer Science, vol. 305 (2003), nos. 1-3, pp. 347-408.

[98] — The Basic Picture: Structures for Constructive Topology, Oxford Logic Guides, Clarendon Press, Oxford, 2018.

[99] T. SANDQvist, Preservation of structural properties in intuitionistic extensions of an inference relation, this JOURNAL, vol. 24 (2018), no. 3, pp. 291-305.

[100] K. Schlagbauer, P. Schuster, and D. Wessel, Der Satz von Hahn-Banach per Disjunktionselimination. Confluentes Mathematici, vol. 11 (2019), no. 1, pp. 79-93.

[101] D. ScotT, On engendering an illusion of understanding. The Journal of Philosophy, vol. 68 (1971), pp. 787-807.

[102] - Completeness and axiomatizability in many-valued logic, Proceedings of the Tarski Symposium (L. Henkin, J. Addison, C. C. Chang, W. Craig, D. Scott, and R. Vaught, editors), Proceedings of Symposia in Pure Mathematics, Vol. XXV, American Mathematical Society, Providence, 1974, pp. 411-435.

[103] D. S. ScotT, Background to formalization, Truth, Syntax and Modality (H. Leblanc, editor), Studies in Logic and the Foundations of Mathematics, Vol. 68, North-Holland, Amsterdam, 1973, pp. 244-273. 
[104] D. J. Shoesmith and T. J. Smiley, Multiple-Conclusion Logic, Cambridge University Press, Cambridge, 1978.

[105] G. Takeuti, Proof Theory, second ed., Studies in Logic and the Foundations of Mathematics, vol. 81, North-Holland Publishing Co., Amsterdam, 1987.

[106] A. TARsKI, Fundamentale Begriffe der Methodologie der deduktiven Wissenschaften.

I. Monatshefte für Mathematik Physik, vol. 37 (1930), pp. 361-404.

[107] A. S. Troelstra and H. Schwichtenberg, Basic Proof Theory, second edition, Cambridge University Press, Cambridge, 2000.

[108] A. S. Troelstra and D. van Dalen, Constructivism in Mathematics: An Introduction, Studies in Logic and the Foundations of Mathematics, vol. I, North-Holland, Amsterdam, 1988.

[109] A. S. Troelstra, On the early history of intuitionistic logic, Mathematical Logic (P. P. Petkov, editor), Plenum Press, New York, 1990, pp. 3-17.

[110] M. van Atten and G. Sundholm, L.E.J. Brouwer's 'Unreliability of the Logical Principles': A new translation, with an introduction. History and Philosophy of Logic, vol. 38 (2017), no. 1, pp. 24-47.

[111] D. van Dalen, Logic and Structure, fifth ed., Universitext, Springer, London, 2013.

[112] S.-M. WANG and P. Cintula, Logics with disjunction and proof by cases. Archive for Mathematical Logic, vol. 47 (2008), no. 5, pp. 435-446.

[113] D. WESSEL, Choice, extension, conservation. From transfinite to finite proof methods in abstract algebra, Ph.D. thesis, Università degli Studi di Trento, 2018.

[114] - Ordering groups constructively. Communications in Algebra, vol. 47 (2019), no. 12 , pp. $4853-4873$.

[115] _ Point-free spectra of linear spreads, Mathesis Universalis, Computability and Proof (S. Centrone, S. Negri, D. Sarikaya, and P. Schuster, editors), Synthese Library, Springer, Cham, 2019, pp. 353-374.

[116] - A note on connected reduced rings. Journal of Commutative Algebra, vol. 13 (2021), no. 4, pp. 583-588.

[117] R. WóJCICKI, Theory of Logical Calculi: Basic Theory of Consequence Operations, Synthese Library, vol. 199, Kluwer Academic Publishers Group, Dordrecht, 1988.

[118] I. Yengui, Making the use of maximal ideals constructive. Theoretical Computer Science, vol. 392 (2008), pp. 174-178.

[119] - Constructive Commutative Algebra: Projective Modules over Polynomial Rings and Dynamical Gröbner Bases, Lecture Notes in Mathematics, vol. 2138, Springer, Cham, 2015.

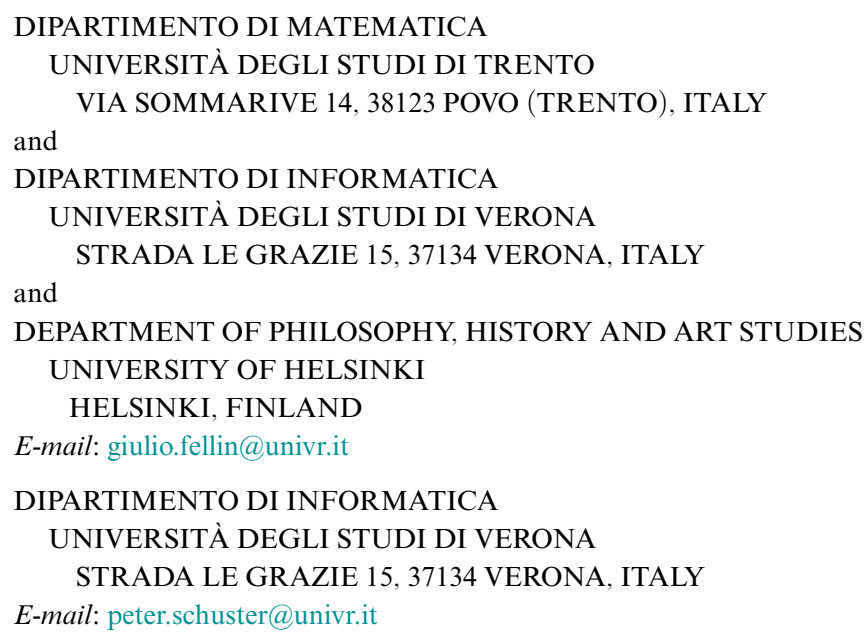


DIPARTIMENTO DI INFORMATICA

UNIVERSITÀ DEGLI STUDI DI VERONA

STRADA LE GRAZIE 15, 37134 VERONA, ITALY

and

MATHEMATISCHES INSTITUT DER UNIVERSITÄT MÜNCHEN

THERESIENSTR. 39, D-80333 MÜNCHEN, GERMANY

E-mail: daniel.wessel@univr.it 